\title{
Direct observation of internal vortex domain-wall dynamics
}

\author{
Falk-Ulrich Stein, ${ }^{1, *}$ Lars Bocklage, ${ }^{1,2,3}$ Markus Weigand, ${ }^{4}$ and Guido Meier ${ }^{1,3}$ \\ ${ }^{1}$ Institut für Angewandte Physik und Zentrum für Mikrostrukturforschung, Universität Hamburg, Jungiusstrasse 11, 20355 Hamburg, Germany \\ ${ }^{2}$ Deutsches Elektronen-Synchrotron, Notkestrasse 85, 22607 Hamburg, Germany \\ ${ }^{3}$ The Hamburg Centre for Ultrafast Imaging, Luruper Chaussee 149, 22761 Hamburg, Germany \\ ${ }^{4}$ Max-Planck-Institut für Intelligente Systeme, Heisenbergstrasse 3, 70569 Stuttgart, Germany
}

(Received 17 September 2013; revised manuscript received 9 December 2013; published 30 January 2014)

\begin{abstract}
The motion of domain walls is influenced by their internal structure and their structural changes during motion. The macroscopic motion is well understood but the internal dynamics during motion have not been experimentally studied in detail. We study vortex domain walls excited by nanosecond long magnetic field pulses in a pinning potential and above the pinning threshold. The dynamic response is imaged by transmission $\mathrm{x}$-ray microscopy and the structural changes are analyzed. From the directly observed inertial behavior of the wall the domain-wall mass equivalent is estimated. During motion, oscillations of both the vortex core around the center of the domain wall and the domain-wall width are observed. The wall shows a fully elastic behavior by compression and overexpansion when being pushed by the field.
\end{abstract}

DOI: 10.1103/PhysRevB.89.024423

PACS number(s): 75.60.Ch, 75.78.Fg, 85.70.-w

Magnetic domain walls in flat nanowires possess unique properties [1]. Although having a rather complex magnetization structure they are, to a large extent, describable as quasiparticles [2]. A controlled motion of these walls can be achieved by magnetic fields [3] and electric currents [4,5]. The equations of motion are first-order differential equations [6] compared to second-order differentials for classical particles. But like classical particles an inertial behavior of domain walls is expected [7] and has been observed for domain walls in nanowires [8-10]. This inertial behavior is expressed by an effective mass of the domain wall $[7,8,11]$ that only exist for walls in motion. Their controllable motion under the influence of spin polarized currents and magnetic fields have led to several ideas for applications like nonvolatile memory, logic devices, and sensors [12-14]. In general, two types of domainwall motion exist. For low driving forces the walls move as almost rigid particles, while above the Walker breakdown internal dynamics govern the macroscopic motion [2]. The velocity is proportional to the driving force up to the Walker breakdown [2] and the walls move slower for forces just above it. Measurements have shown a good agreement of this behavior between the theoretical description and the macroscopic motion $[3,15,16]$. However, most experiments so far had no direct access to the internal dynamics of the domain walls that can have strong impact on their behavior. Time-resolved $\mathrm{x}$-ray microscopy has been used to study domain-wall oscillations in confined potentials [11,17] and the fast generation of vortex domain walls [10]. The driven motion of the walls has only been observed in strong fields above the Walker breakdown [10] as strong Oersted field pulses are required for the fast generation of domain walls $[18,19]$. For applications a detailed understanding of the dynamics in lower driving forces is crucial. We present time-resolved $\mathrm{x}$-ray microscopy of internal dynamics of vortex domain walls under the influence of magnetic field pulses. Oscillations of the wall width as well as of the vortex core are observed. The domain-walls internal structural changes correlate with

*fstein@physnet.uni-hamburg.de the macroscopic motion of the wall. In addition, the excitation of walls just below the pinning threshold is analyzed.

Kinked permalloy nanowires are suitable for controlled injection of vortex domain walls by external magnetic fields [20]. By widening the wire in the direction away from the kink a confining potential for the wall is generated as the domainwall energy is proportional to the wire width [21]. We use $350-430 \mathrm{~nm}$ wide and $20 \mathrm{~nm}$ thick permalloy $\left(\mathrm{Ni}_{80} \mathrm{Fe}_{20}\right)$ wires prepared on silicon-nitride membranes for the direct observation of internal dynamics of the wall during motion. Imaging is done by scanning transmission X-ray microscopy [22] with the MAXYMUS microscope at BESSY II synchrotron with a spatial resolution of $25 \mathrm{~nm}$ given by the Fresnel zone plate used in this experiment and a temporal step size of $400 \mathrm{ps}$. The nanowires were fabricated by electron-beam lithography and thermal evaporation on silicon covered silicon-nitride membranes. After a second lithography step the stripline was deposited by sputter deposition of an aluminum/gold contact layer and thermal evaporation of $90 \mathrm{~nm}$ copper and $8 \mathrm{~nm}$ of gold. Figure 1(a) shows the measurement setup with a kinked wire and a $2 \mu \mathrm{m}$ wide stripline. After saturation of the wire with a perpendicular external magnetic field $H_{\text {sat }}$ along the $y$ direction a domain wall is created next to the kink. Due to opposite angles of the arms of the wire with respect to the magnetic field $H_{\text {sat }}$ the magnetization relaxes into opposite directions when the field is switched off, thus forming a vortex domain wall in these wire dimensions. A current pulse generated by a pulse generator is injected into the stripline. The Oersted field underneath the stripline is aligned almost parallel to the magnetization in the lower arm and pushes the domain wall away from the kink. After the field pulse is over the domain wall is driven back into its initial position by the force resulting from the potential of the widening wire. For the purpose of time-resolved imaging this process is repeated at $16 \mathrm{MHz}$ synchronized to the $\mathrm{x}$-ray flashes in an asynchronous pump and probe scheme [10]. Figure 1(b) shows a domain wall that is moved upwards by a field pulse. The domain wall is accelerated upwards and gets distorted. After the field pulse ends the wall slows down and stops at $7.5 \mathrm{~ns}$. The potential from the widening wire then leads to a backwards motion of the 

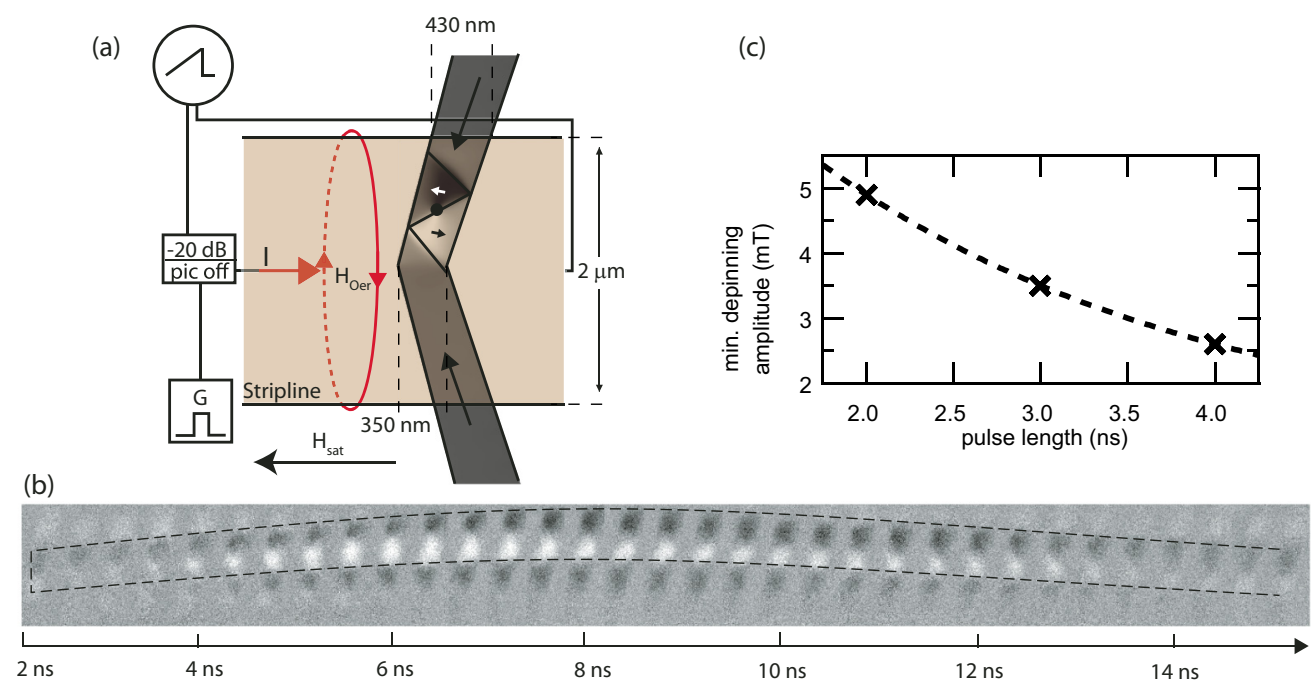

FIG. 1. (Color online) (a) Experimental setup with a $2 \mu \mathrm{m}$ wide stripline across a kinked permalloy nanowire. An external magnetic field $H_{\text {sat }}$ is used to create a vortex domain wall next to the kink that is excited by the Oersted field $H_{\text {Oersted }}$ of a current pulse. The ingoing $(-20 \mathrm{~dB}$ pick off) and outgoing pulses are monitored by an oscilloscope. (b) Time-resolved sequence of scanning transmission x-ray micrographs of an excited domain wall over a period of $15 \mathrm{~ns}$. (c) Measured minimal field pulse strength (crosses) for depinning a domain wall from the kink in dependence of the pulse length and an exponentially decaying fit (dashed black line).

domain wall restoring the initial magnetization configuration. In the beginning a white/black/white contrast is observed. While the lower white/black part shows the resting domain wall next to the kink the top white area is a residue from normalization of the images. This is also the case for the lower dark contrast from 4 until 12 ns where the white contrast area of the resting domain wall results in a dark shadow when the domain wall moves upwards. The normalization also results in the contrast being strongest when the domain wall is at its turning point. The strength of the field pulses is calculated by numerical integration of Biot-Savarts law using the transmitted voltage signal measured by the oscilloscope. The minimum field strengths that are needed to actually move the domain wall away from the kink are shown in Fig. 1(c). As the force of the field on the domain wall acts longer the required depinning field decreases. If the field pulse duration and amplitude are too low to depin the domain wall from the resting position at the kink the wall gets excited in a nearly harmonic potential [23,24]. In Figs. 2(a) and 2(b) the position of the vortex core of the domain walls over time is shown for pulses of 2 and 3 ns length for various field amplitudes. The position is gained from the x-ray micrographs by determination of the inflection point of a polygonal fit through the black/white contrast. Two types of behaviors are observed for both pulse lengths. For 2 ns long pulses below $5 \mathrm{mT}$ and for $3 \mathrm{~ns}$ long pulses below $3.6 \mathrm{mT}$ an oscillation of the domain wall around the pinned position is observed. The amplitude of this oscillation changes only slightly for different excitation amplitudes. While the $3 \mathrm{~ns}$ long pulse is applied from 2 until 5 ns the wall is forced into a deflected position and starts a free oscillation at the pinning site after the pulse is over. A fit of the damped oscillation $X(t)=X_{0}+$ $A \cos \left[\omega\left(t-t_{0}\right)\right] e^{-\Gamma\left(t-t_{0}\right)}$ in Fig. 2(a) gives an eigenfrequency of the pinned domain wall of $\omega=980( \pm 25) \mathrm{Mrad} / \mathrm{s}$ with a damping constant of $\Gamma=150( \pm 27) \mathrm{MHz}$.
When the amplitude is increased above the depinning threshold the wall moves away from the kink and continues the outward motion beyond the end of the pulse. The $2 \mathrm{~ns}$ long pulse ends at $4 \mathrm{~ns}$ in the measurement shown in Fig. 2(a). The wall only gradually slows down until the turning point is reached $2.7 \mathrm{~ns}$ later. The same can be observed for $3 \mathrm{~ns}$ long

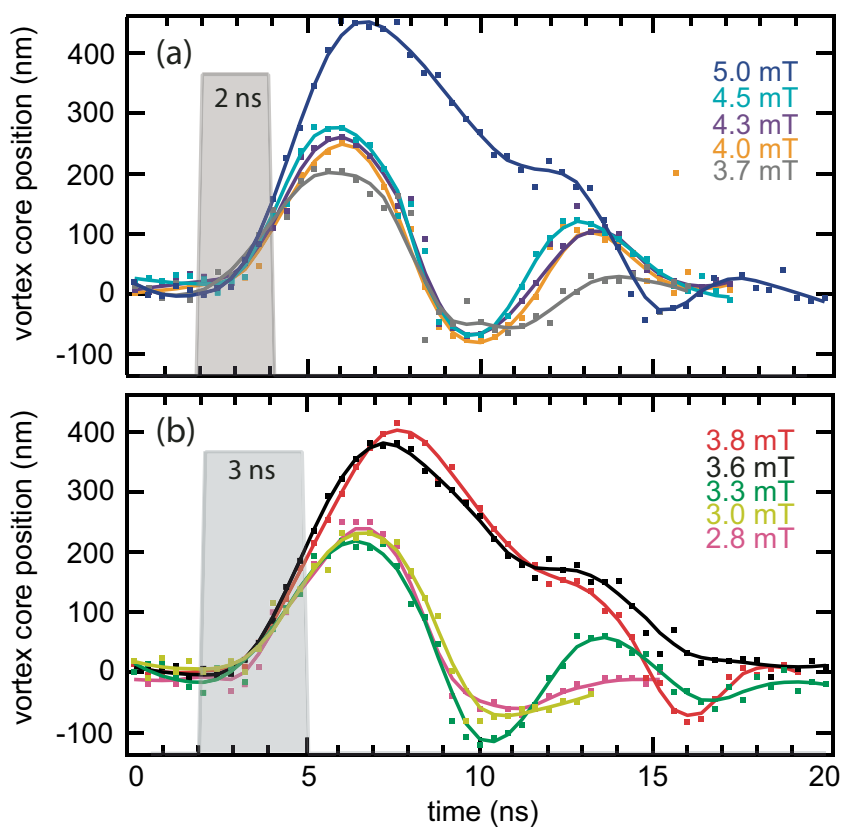

FIG. 2. (Color online) Measured position of the vortex core of a domain wall excited by (a) 2 ns and (b) 3 ns long field pulses for various field strengths. The domain wall either stays pinned and oscillates or is depinned and moves outwards until the field pulse ends and the restoring potential returns it to its initial position. The solid lines are guides to the eye. 

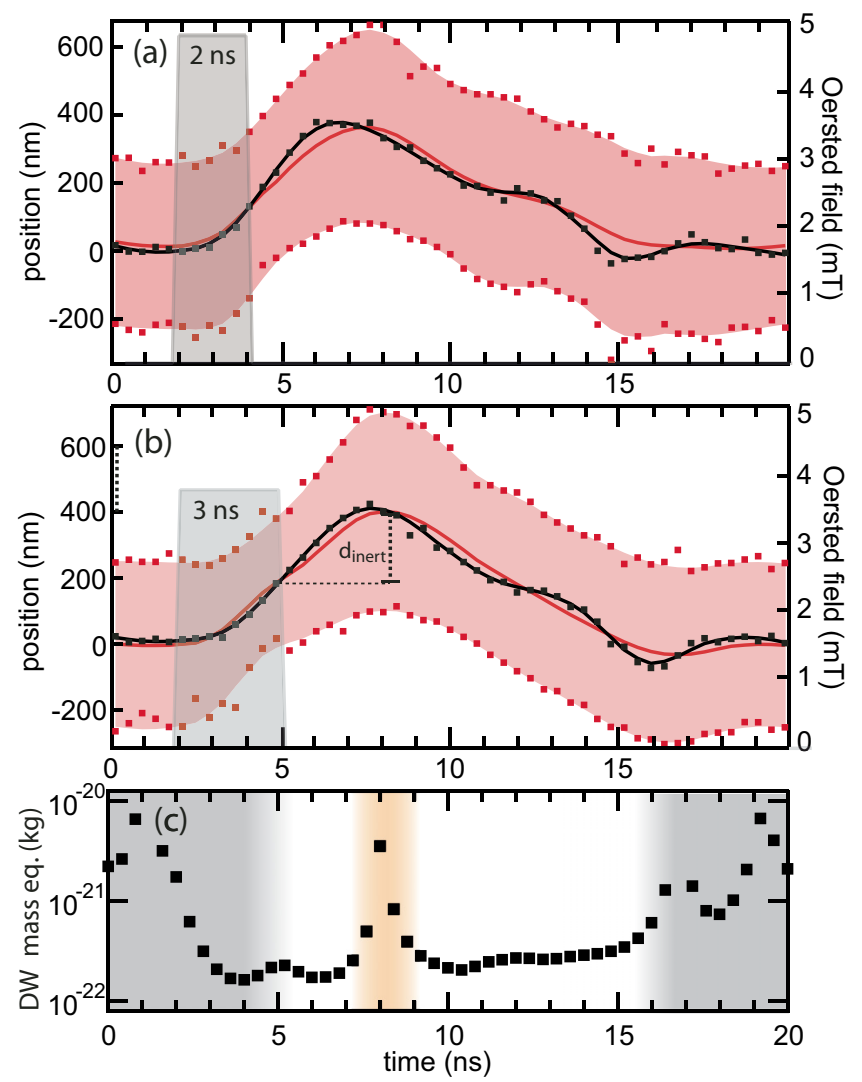

FIG. 3. (Color online) Positions of depinned domain walls over time after excitement by (a) $2 \mathrm{~ns}$ long and (b) $3 \mathrm{~ns}$ long field pulses. The position of the vortex core is shown in black, the averaged center of the domain wall in solid red. The red dots show the upper and lower edge of the domain wall. The strength of the pulse is plotted on the right and the position on the left axis. The domain-wall mass equivalent is shown over time in (c). In the shaded areas the applied definition is not valid.

pulses where the pulse ends at $5 \mathrm{~ns}$ in Fig 2(b). At fields of 3.6 and $3.8 \mathrm{mT}$ the motion continues for another 2.3 and $2.7 \mathrm{~ns}$ before moving back to its equilibrium position, respectively. This is a direct observation of the inertial behavior [9] of domain walls in nanowires on the intrinsic time and length scale of the process. After the turning point is reached at $7.5 \mathrm{~ns}$ the encountering potential moves the wall back towards the kink. The velocity of $70 \mathrm{~m} / \mathrm{s}$ on the return is lower compared to the case where the wall is pushed by the field pulse with $120 \mathrm{~m} / \mathrm{s}$. Between 10 and $20 \mathrm{~ns}$ the backward motion of the depinned walls shows a slight oscillation for both 2 and $3 \mathrm{~ns}$ long pulses. This is not an oscillation of the whole wall but only of the vortex core. The edges of the domain wall do not show the same behavior. In Figs. 3(a) and 3(b) the upper and the lower edges of the wall are shown (red dotted lines). The position of the transition between the black and the white parts of the domain wall is the vortex core position (black line). The center of the domain wall (red line) being the average of the upper and the lower edge moves with constant velocity while the vortex core (black line) oscillates around the averaged wall center. For both pulse lengths it is visible that the vortex core reaches the turning point $0.7 \mathrm{~ns}$ before the averaged center of the wall although the vortex core has a higher velocity at the end of the pulse. This delay indicates that the inertia of the vortex core is lower than that of the averaged center. The latter also does not reach the same maximum velocity as the vortex core. Compared to the maximum velocity of $120 \mathrm{~m} / \mathrm{s}$ for the pushed vortex core the averaged center of the wall only reaches $95 \mathrm{~m} / \mathrm{s}$. The equations of motion for a transverse domain wall [6,11] from the one-dimensional (1D) model differ from the classical description of a moving particle by including a factor depending on the velocity of the wall $v$ and its damping time $\tau_{d}$. In previous work we have shown that the model is also capable of describing the macroscopic motion of vortex domain walls as well when adapted parameters are used [10,25]. The mass equivalent in the 1D model for motion in a potential $E$ without an external magnetic field is

$$
m_{1 \mathrm{D}}^{\prime}=-\frac{d E}{d x} \frac{1}{\frac{v}{\tau_{d}}+a},
$$

with the acceleration $a$, the velocity $v$, and the damping time $\tau_{d}$. The latter can be gained from the damping of the free oscillation in Fig. 2(a) by $\tau_{d}=(2 \Gamma)^{-1}=3.3 \mathrm{~ns}[11,26]$. From micromagnetic simulations the slope of the potential is found to be $\frac{d E}{d x}=-4.7 \times 10^{-12} \mathrm{~J} / \mathrm{m}$ [27]. Figure 3(c) shows the domain-wall mass over time gained from the data of the averaged domain-wall position in Fig. 3(b). For the stationary moving domain wall (nonshaded area) the mass is almost constant with an average value of $2.3( \pm 0.1) \times 10^{-22} \mathrm{~kg}$. The gray shaded areas mark where Eq. (1) is not valid as there is either a field present or the domain wall is inside the pinning potential near the kink. At the turning point of the wall the mass equivalent also increases by two orders of magnitude (orange shaded area). This increase is most likely due to strong deformations of the wall that are present at the turning point and are discussed later. However, the description within the one-dimensional model is only applicable for small deformations [6]. The constant mass during stationary motion in the present work shows that the model description is reasonable even though the domain wall is not a fully rigid particle. The change of the domain-wall mass reflects the internal dynamics. The mass equivalent of the domain wall can also be estimated from the inertia of the wall after the pulse in direct comparison to a classical particle that is stopped by a constant force. The average deceleration after the pulse can be calculated from the maximum velocity $v_{\max }=95( \pm 15) \mathrm{m} / \mathrm{s}$ and the distance traveled under inertial motion $d_{\text {inert }}=212( \pm 40) \mathrm{nm}$ to $a=\frac{v_{\max }^{2}}{2 d_{\text {inert }}}=2.1( \pm 1.1) \times 10^{10} \mathrm{~m} / \mathrm{s}^{2}$. A classical calculation of the mass of $m_{\text {classic }}=-\frac{d E}{d x} \frac{1}{a}=2.2( \pm 1.1) \times 10^{-22} \mathrm{~kg}$ is in surprisingly good agreement with our calculations with the adapted 1D model in this work and a previous publication [10] as well as theoretical predictions [29]. As expected for an estimate the error is large compared to the model calculations.

The red areas in Fig. 3 are representing the width of the domain wall that changes during the excitation. While the pulse is applied the lower edge of the domain wall is pushed to the center of the wall. This results in a compression of the wall. In Fig. 4(a) the width of the domain walls is shown versus time for the $2 \mathrm{~ns}$ long (blue line and blue pulse) and for the 3 ns long pulses (black and red lines and gray pulse). The resting wall is $500 \mathrm{~nm}$ wide and gets compressed to $470 \mathrm{~nm}$ 


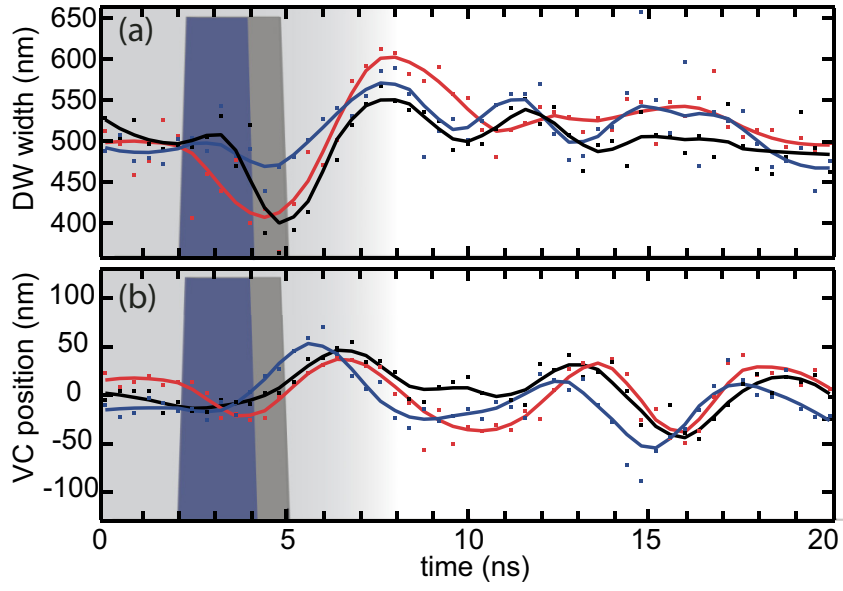

FIG. 4. (Color online) (a) Width of depinned domain walls excited by 2 ns long (blue) and 3 ns long pulses (red, black). The pulses are indicated by the gray and blue shaded areas. (b) Position of the vortex core for the same domain walls. The shaded area indicates the outward motion until the turning point.

by $2 \mathrm{~ns}$ long pulses and to $400 \mathrm{~nm}$ by $3 \mathrm{~ns}$ long pulses. The compression is followed by an overexpansion up to $600 \mathrm{~nm}$ and keeps oscillating afterwards. Figure 4(b) shows the position of the vortex core relative to the averaged center of the wall during the same time span. The core seems to be slightly expelled to the bottom by the pulse around $4 \mathrm{~ns}$. This is not a motion of the vortex core outwards but follows from the compression of the wall seen in Figs. 3(b) and 4(a). Initially the lower end of the wall gets pushed in thereby shifting the averaged center of the wall towards the top while the vortex core is accelerated later. When the wall is compressed further the averaged center is also pushed further upwards. The strong compression of the lower domain-wall part results in a delayed deflection of the vortex core of $50 \mathrm{~nm}$ from the center at around $6 \mathrm{~ns}$, thereby overtaking the averaged center of the wall. From 8 to $10 \mathrm{~ns}$ the oscillation of the vortex core seems to pause as the domain wall had reached the turning point. The vortex core then oscillates around the averaged center during a steady backwards motion of the domain wall. The oscillation frequency of $214( \pm 3) \mathrm{MHz}$ is comparable to the eigenfrequency of a vortex in an ellipse of the same size as the domain wall [17]. The oscillation appears not to be damped but the observed time length might not be long enough to identify a low damping. Oscillations at similar frequencies have been found for continuously field driven domain walls in linear wires [15]. Since the velocity of the wall on its return reaches $70 \mathrm{~m} / \mathrm{s}$ (compared to $95 \mathrm{~m} / \mathrm{s}$ when field driven) it is possible that the oscillation of the vortex core around the averaged center of the wall is due to approaching the Walker breakdown.

In Fig. 5(a) the widths of the upper (black line) and the lower part (gray line) of the domain wall with respect to the vortex core are plotted as a function of time. With the beginning of the pulse the lower half gets compressed first and is followed by a slight compression of the upper half with a phase shift of $1.3 \mathrm{~ns}$ corresponding to $\frac{\pi}{2}$ of its eigenfrequency. $2 \mathrm{~ns}$ after the turning point the lower part oscillates undisturbed with slightly changing frequency, while the upper half's oscillation

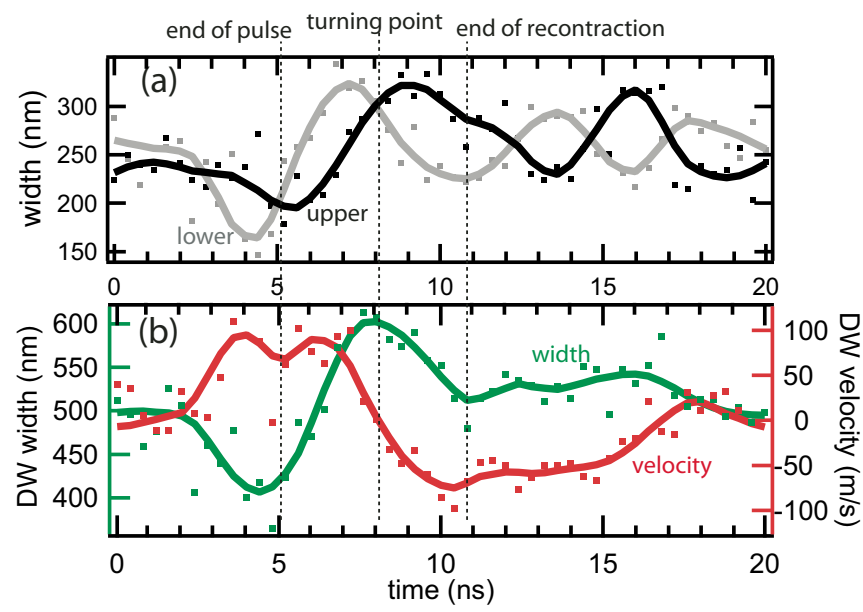

FIG. 5. (Color online) (a) Widths of the upper (black line) and lower (gray line) half of the domain wall shown in red in Fig. 3. (b) Velocity (red line) and total width (green line) of the domain wall during the motion. The width is plotted on the left and the velocity on the right axis.

is disturbed to enter a $\pi$-phase shifted motion. This $\pi$-phase shifted oscillation of the upper and lower half of the domain wall shows the oscillation of the vortex core around the center of the wall while retaining its width as seen in Fig. 4(a). Figure 5(b) shows the velocity of the wall together with the total wall width gained from the position data in Fig. 3(b). The disturbance of the wall motion at $11 \mathrm{~ns}$ occurs by the time the maximum negative velocity is reached. The pulse, that starts at $2 \mathrm{~ns}$, compresses the wall and accelerates the averaged wall center for $2 \mathrm{~ns}$ reaching $90 \mathrm{~m} / \mathrm{s}$. After the end of the pulse at $5 \mathrm{~ns}$ the wall expands reaching its maximum expansion at the turning point ( $8 \mathrm{~ns})$. After the wall has stopped it is accelerated into the opposite direction accompanied by the relaxation of the wall width until $11 \mathrm{~ns}$. At $11 \mathrm{~ns}$ the velocity slightly decreases when the expanded wall has relaxed to its original size. A maximum negative velocity of $70 \mathrm{~m} / \mathrm{s}$ is reached and the wall moves with an almost constant velocity. The vortex core oscillates around the center as seen in Fig. 4(b). This analysis shows the interplay of the internal dynamics of the domain wall and its macroscopic motion. The domain wall is compressed during acceleration by the field pulse. The vortex core is deflected from the averaged domain-wall center due to the compression. The following expansion and recontraction of the wall concur with changes in the velocity of the wall for example at the turning point. On the return to its initial position driven by the linear potential the domain wall moves without modulations of its width. The vortex core again oscillates around the averaged wall center before the wall is pinned near the kink in the nanowire.

In summary, we present the time-resolved imaging of the field pulse driven dynamics of a pinned and depinned vortex domain wall. Below the depinning threshold the wall oscillates at its eigenfrequency. During depinned motion the dynamics of the domain wall are rather complex with oscillations of the total width of the wall as well as of the vortex core around the averaged center of the wall. Scanning transmission x-ray microscopy gives access to direct observation of these internal 
dynamics of the wall. These changes in width concur with changes of the macroscopic motion of the domain wall. The observations show the phase shift in acceleration of the vortex core as well as the upper and lower edge of the domain wall. From the analysis of stationary motion and the slowdown of the wall after the pulse end we determine the domain-wall mass and show that the inertia of the vortex core is smaller than of the averaged center of the domain wall. During the linear motion to its initial position an oscillation of the vortex core around the center of the wall is observed without perceptible damping.

[1] C. H. Marrows and G. Meier, J. Phys.: Condens. Matter 24, 020301 (2012).

[2] N. L. Schryer and L. R. Walker, J. Appl. Phys. 45, 5406 (1974).

[3] G. S. D. Beach, C. Nistor, C. Knutson, M. Tsoi, and J. L. Erskine, Nat. Mater. 4, 741 (2005).

[4] G. Tatara and H. Kohno, Phys. Rev. Lett. 92, 086601 (2004).

[5] A. Yamaguchi, T. Ono, S. Nasu, K. Miyake, K. Mibu, and T. Shinjo, Phys. Rev. Lett. 92, 077205 (2004).

[6] B. Krüger, D. Pfannkuche, M. Bolte, G. Meier, and U. Merkt, Phys. Rev. B 75, 054421 (2007).

[7] W. Döring, Z. Naturforsch. Teil A 3, 373 (1948).

[8] E. Saitoh, H. Miyajima, T. Yamaoka, and G. Tatara, Nature (London) 432, 203 (2004).

[9] L. Thomas, R. Moriya, C. Rettner, and S. S. P. Parkin, Science 330, 6012 (2010).

[10] F.-U. Stein, L. Bocklage, M. Weigand, and G. Meier, Sci. Rep. 3, 1737 (2013).

[11] L. Bocklage, B. Krüger, R. Eiselt, M. Bolte, P. Fischer, and G. Meier, Phys. Rev. B 78, 180405(R) (2008).

[12] S. S. P. Parkin, M. Hayashi, and L. Thomas, Science 320, 190 (2008).

[13] D. A. Allwood, G. Xiong, C. C. Faulkner, and D. Atkinson, D. Petit, and R. P. Cowburn, Science 309, 1688 (2005).

[14] R. Mattheis, S. Glathe, M. Diegel, and U. Hübner, J. Appl. Phys. 111, 113920 (2012).

[15] M. Hayashi, L. Thomas, C. Rettner, R. Moriya, and S. S. P. Parkin, Nat. Phys. 3, 21 (2007).

[16] J. Yang, C. Nistor, G. S. D. Beach, and J. L. Erskine, Phys. Rev. B 77, 014413 (2008).
We thank Ulrich Merkt for persistent support and fruitful discussions and Christian Adolff, Toru Matsuyama, Michael Volkmann, and Michael Bechtel for excellent technical support. Financial support by the Deutsche Forschungsgemeinschaft via Sonderforschungsbereich 668 and via Graduiertenkolleg 1286 is gratefully acknowledged. The measurements were conducted at the MAXYMUS microscope of the Max Planck Institut für Intelligente Systeme at beamline UE46-PGM2 at BESSY II, Helmholtz Zentrum Berlin.

[17] A. Bisig, J. Rhensius, M. Kammerer, M. Curcic, H. Stoll, G. Schütz, B. Van Waeyenberge, K. W. Chou, T. Tyliszczak, L. J. Heyderman et al., Appl. Phys. Lett. 96, 152506 (2010).

[18] F.-U. Stein, L. Bocklage, T. Matsuyama, and G. Meier, App. Phys. Lett. 100, 192403 (2012).

[19] L. Bocklage, F.-U. Stein, M. Martens, T. Matsuyama, and G. Meier, Appl. Phys. Lett. 103, 092406 (2013).

[20] S. Hankemeier, A. Kobs, R. Frömter, and H. P. Oepen, Phys. Rev. B 82, 064414 (2010).

[21] R. D. McMichael and M. J. Donahue, IEEE Trans. Mag. 33, 4167 (1997).

[22] H. Ade and H. Stoll, Nat. Mater. 8, 281 (2009).

[23] R. Moriya, L. Thomas, M. Hayashi, Y. B. Bazaliy, C. Rettner, and S. S. P. Parkin, Nat. Phys. 4, 368 (2008).

[24] C. Wuth, L. Kolbe, and G. Meier, J. Appl. Phys. 114, 103901 (2013).

[25] L. Bocklage, B. Krüger, T. Matsuyama, M. Bolte, U. Merkt, D. Pfannkuche, and G. Meier, Phys. Rev. Lett. 103, 197204 (2009).

[26] L. Bocklage, B. Krüger, P. Fischer, and G. Meier, Phys. Rev. B 81, 054404 (2010).

[27] The simulations are carried out with Micromagnum [28] using a cell size of $5 \times 5 \times 5 \mathrm{~nm}^{3}$, a saturation magnetization of $8.5 \times 10^{5} \mathrm{~A} / \mathrm{m}$, and a damping of $\alpha=0.01$. The simulated geometries are the same as that of the measured sample. In order to determine the potential slope a vortex wall is relaxed at the outer end of the structure and the total energy of the system is calculated during the inwards motion of the domain wall.

[28] MicroMagnum Fast Physical Simulator for Computations on CPU and Graphics Processing Unit http://micromagnum.informatik.uni-hamburg.de/.

[29] D. J. Clarke, O. A. Tretiakov, G.-W. Chern, Y. B. Bazaliy, and O. Tchernyshyov, Phys. Rev. B 78, 134412 (2008). 